\title{
HIGHER DERIVATIONS AND AUTOMORPHISMS OF COMPLETE LOCAL RINGS
}

\author{
BY NICKOLAS HEEREMA
}

ABSTRACT. This paper begins with a review of those aspects of the theory of higher derivations on fields which form a background for the study of recent uses of higher derivations in automorphism theory of complete local rings. Basic definitions and basic properties of convergent higher derivations on complete local rings are discussed including the concept of convergent rate group of automorphisms, the theory of which is at the present time almost totally undeveloped.

Methods of constructing automorphisms using higher derivations are considered next, particularly in connection with the problem of identifying the factor groups of the higher ramification series of a complete local ring. Recent results on this problem are discussed as well as some possible directions for future research on the topics of this article.

\section{Table of Contents}

I. Introduction. ....................... 1212

II. Basic definitions and basic properties.......... 1213

III. Construction of higher derivations............ 1215

IV. Derivation automorphisms, convergence rate automorphisms and the ramification series............ 1217

V. Approximating inertial automorphisms with derivation automorphisms......................... 1219

VI. A more versatile method of approximating. ....... 1221

VII. Some applications and some problems.......... 1222

I. Introduction. My purpose in this article is to provide a selective outline of the development of the theory of higher derivations leading to applications in the automorphism theory of complete local rings. As a result certain recent developments in higher derivation theory, except perhaps for casual reference, are outside the scope of this paper, e.g., applications to Galois theory of fields [5], [6], [21], [35], [38] as well as the theory of universal higher derivations and in-

An invited address delivered to the 669th meeting of the Society at Baton Rouge, Louisiana on November 22, 1969; received by the editors May 25, 1970.

AMS 1969 subject classifications. Primary 1360, 1395; Secondary 1393, 1660.

Key words and phrases. Derivation, higher derivation, derivation automorphism, inertial automorphism, convergent rate group, v-ring, complete regular local ring, ramification, ramification series, $p$-basis. 
separability criteria [2], [33]. Proofs, or indications of proof, will be provided now and then to clarify the subject for the general reader.

Let $A$ be an (non-associative) algebra over a field $k$ of characteristic zero and let $d$ be a derivation on $A$, that is, $d$ is an additive map of $A$ into $A$ which satisfies the product rule, $d(a b)=a d(b)+d(a) b$. We will use Der $A$ to denote the additive group of all derivations on $A$. The sequence of maps $\left\{d^{i} / i !\right\}$ (all sequences and sums, unless otherwise indicated, are indexed from 0 to $\infty$ ) is an example of a higher derivation on $A$. If $A$ is complete in some topology in which the above sequence of maps converges then $\exp d=\sum\left(d^{i} / i !\right)$ will be an automorphism on $A$. For example, if $k$ is the field of real numbers and $A$ has finite dimension over $k$ then $k$ is complete in the Euclidean topology and $\exp d$ is a convergent series and hence an automorphism, for all $d$ in Der $A$. Or, in the general case, if $d$ is associative nilpotent then $\exp d$ contains only a finite number of non-zero terms and is thus an automorphism. Such automorphisms have an important role in the structure theory of Lie algebras over fields of characteristic zero [27, Chapter 9].

II. Basic definitions and basic properties.

(1) Definition. Let $h \supset k$ be (non-associative) rings. A set $D$ $=\left\{D^{(i)}\right\}$ of additive maps of $k$ into $h$ which satisfies (i) and (ii) is called a higher derivation of $k$ into $h$.

(i) $D^{(n)}(a b)=\sum\left\{D^{(i)}(a) D^{(n-i)}(b) \mid i=0, \cdots, n\right\}, n \geqq 0$.

(ii) $D^{(0)}=$ Id, (identity map on $k$ ).

Property (i) is called the Leibniz rule. The more familiar Leibniz rule for derivations, namely,

$$
d^{n}(a b)=\sum\left(\begin{array}{l}
n \\
i
\end{array}\right) d^{i}(a) d^{n-i}(b),
$$

allows one to establish (i) in the case of the above example simply by dividing both sides by $n$ !

The above definition was first given by F. K. Schmidt in 1937 [10, p. 224] generalizing a concept first introduced, a year earlier, by $\mathrm{H}$. Hasse in connection with the differential calculus of algebraic curves over fields of prime characteristic, [9], [37]. We shall need a pair of results found originally in the Hasse and Schmidt paper $[10$, pp. 229, 230]. Let $\mathfrak{F}(k, h)$ be the set of all higher derivations on the ring $k$ into the overring $h$. If $k=h$ we abbreviate the notion to $\mathfrak{H C}(k)$.

(2) Proposition. Let $D$ be in $\Re(k, h)$ where $k$ is a subfield of the field $h$, and let $x$ be an element of $h$. If $x$ is transcendental over $k$, then, 
given $\left\{u_{i} \mid i \geqq 1, u_{i} \in h\right\}$, there exists exactly one extension $D_{*}$ in $\mathcal{F C}(k(x), h)$ of $D$ for which $D_{*}^{(i)}(x)=u_{i}, i \geqq 1$. If $x$ is separable algebraic over $k$ then $D$ extends uniquely to $D_{*}$ in $\Re(k(x), h)$.

One can prove the first assertion simply by constructing $D_{*}$ on $k[x]$ as dictated by the conditions $D_{*}^{(i)}(x)=u_{i}$, the Leibniz rule and additivity. Uniqueness follows from the construction. As in the case of derivations, a higher derivation defined on an integral domain has a unique extension to the field of quotients.

We sketch a nonstandard sort of proof of a slight generalization of the second statement of the proposition, assuming the characteristic of $k$ different from zero. The reason for doing this is that the method of proof generalizes to progressively more complicated situations leading to a convenient method for constructing higher derivations on a complete regular local ring having residue field characteristic different from zero (Theorem 16) [18, p. 28, Theorem 4].

We assume now that characteristic $k=p \neq 0$. A basic property of $D$ in $\mathfrak{H C}(k, h)$ is the following.

$$
\begin{aligned}
& D^{(i)}\left(a^{p}\right)=0 \quad \text { if } p \nmid i, \\
& D^{(i)}\left(a^{p}\right)=\left[D^{(i / p)}(a)\right]^{p} \quad \text { if } p \mid i \text {. }
\end{aligned}
$$

The above is proved by $p-1$ applications of the Leibniz rule to $D^{(i)}\left(a^{p}\right)$. It follows then that if $n$ is a positive integer

$$
D^{(i)}\left(a^{p n}\right)=0, \quad \text { if } p^{n} \nmid i \text {. }
$$

Assume now that $k_{1}$ is a subfield of $h$ containing $k$ such that $k_{1} / k$ is separable algebraic. Since $k_{1} / k$ is separable in the linear disjoint sense and $k_{1}=k\left(k_{1}^{p}\right)$ it follows that, given a basis $U$ for $k_{1}$ as a vector space over $k$, then $U^{p}=\left\{u^{p} \mid u \in U\right\}$ is also a basis for $k_{1}$ over $k$. Hence, for any positive integer $n, U^{p^{n}}$ is a basis for $k_{1}$ over $k$. For a given integer $j$ we choose $n$ so that $p^{n}>j$. Then, for $a$ in $k_{1}$, $a=\sum a_{i} u_{i}{ }^{n}, a_{i} \in k, u_{i} \in U$, the sum being over a finite subset of $U$. If $D_{*} \in \mathfrak{F}\left(k_{1}, h\right)$ extends $D$ then

$$
D_{*}^{(j)}(a)=\sum D^{(j)}\left(a_{i}\right) u_{i}^{p^{n}}
$$

by virtue of (3). The desired result is now obtained from an analysis of (4) which clearly implies that if there is a $D_{*}$ extending $D$ there is but one such. Moreover, (4) provides a way of constructing $D_{*}$. It is a routine matter to verify that the maps $D_{*}^{(j)}$ constructed using (4) are independent of the choice of $n$ and that $\left\{D_{*}^{(p)}\right\}$ is a higher derivation extending $D$ (for this it is convenient to assume that 1 is in $U$ ).

Let $k[[X]]$ be the power series ring in a single indeterminate $X$ over the field $k$. We consider the group $g$ of automorphisms on $k[[X]]$ 
defined by $g=\{\alpha \mid \alpha-\operatorname{Id}(k[[X]]) \subset X k[[X]], \alpha(X)=X\}$. The following bijection between $\mathscr{I}$ and $\mathfrak{F C}(k)$ was essentially noted by Schmidt [10]. Given $D$ in $\mathfrak{F C}(k)$ let $\alpha_{D}(\in \mathfrak{g})$ be defined by $\alpha_{D}(a)$ $=\sum D^{(i)}(a) X^{i}$ for $a$ in $k$ and $\alpha_{D}(X)=X$. It is a routine matter to verify that the restriction of $\alpha_{D}$ to $k$ is an isomorphism which takes $a$ into a power series with constant term $a$ and, hence, that the given conditions determine an element of $\mathscr{g}$. Conversely, if $\alpha$ is in $\mathscr{g}$ and $i$ is a nonnegative integer we define $D^{(i)}(a)$ to be $a_{i}$ where $\alpha(a)=\sum a_{i} X^{i}$. Again, it is a simple matter to show that $D^{\alpha}=\left\{D^{(i)}\right\}$ is in $\mathfrak{F C}(k)$ and that $\alpha=\alpha_{D^{\alpha}}$. The bijection $\alpha \rightarrow D^{\alpha}$ induces group structure on $\mathcal{F}(k)$ in the natural way, the group operation " 0 " being given by $D \circ E=F$ where

$$
F^{(n)}=\sum\left\{D^{(i)} E^{(n-i)} \mid i=0, \cdots, n\right\} .
$$

A portent of things to come; we have a group of higher derivations isomorphic to a group of inertial automorphisms of a complete local ring.

Recently, R. L. Davis obtained a generalization of the Jacobson Galois theory for purely inseparable exponent one field extensions to purely inseparable extensions of arbitrary finite exponent $n+1$ [5], [6], [25]. In this theory Davis uses the finite higher derivation $\left\{D^{(i)} \mid i=0, \cdots, p^{n}\right\}$ as a generalization of derivation and the operation " $\circ$ " to generalize addition of derivations. The closure with respect to $p$ th powers condition of the Jacobson theory appears in the Davis theory in the factors of the upper central series of the group of higher derivations, these being additive groups of derivations.

III. Construction of higher derivations. The concept, higher derivation, was originally introduced in order to remove some of the anomalies in the calculus of derivations on fields of characteristic $p \neq 0$ [9], [28]. Nevertheless, higher derivations have important applications in characteristic zero situations, as we shall see. The following result provides considerable information about higher derivations in the latter case.

(5) Theorem [11, pp. 190-191]. Let $A$ be an algebra over a field $k$ having characteristic zero. Given a sequence

$$
\left\{d_{i} \mid d_{i} \in \operatorname{Der} R, i=1, \cdots, \infty\right\},
$$

the sequence $D=\left\{D^{(i)}\right\}$ is in $\operatorname{FC}(A)$ where

$$
D^{(n)}=\sum\left\{\frac{d_{i_{1}} \cdots d_{i_{r}}}{r !} \mid i_{1}+\cdots+i_{r}=n\right\}, \quad n \geqq 1 .
$$


Moreover, the correspondence $\left\{d_{i}\right\} \rightarrow D$ is a bijection between the set of all sequences $\left\{d_{i}\right\}$ and $\mathfrak{H}(A)$.

This theorem is contained in the above reference for the case in which $A$ is a field, however, the proof given there does in fact serve for the result as stated above.

We see by Theorem 5 that the abundance of higher derivations on $A$ is determined by the abundance of derivations. In 1927, R. Baer showed that the subfields of a field $h$ of characteristic zero which are fields of constants of derivations on $h$ are precisely those subfields $k$ algebraically closed in $h[1]$. The field of constants of $D$ in $\Re(h)$ is $h^{D}=\left\{a \in h \mid D^{(i)}(a)=0, i>0\right\}$. Since the intersection of subfields of $h$, each of which is algebraically closed in $h$, is again algebraically closed in $h$ it follows from the above theorem that the fields of constants of higher derivations on $h$ are again the subfields of $h$ algebraically closed in $h$.

The similarity between (6) and our first example of a higher derivation suggests that the map exp may have a more substantial connection with higher derivations than the relationship observed in that example. This is the case and that connection as well as the resulting relationship between the Campbell Hausdorff formula and products of higher derivations has been investigated by the writer [22].

An analog of Theorem 5 in the case in which $A$ is a field having characteristic $p, \neq 0$, is given below (Theorem 9). This result is vital to a technique for constructing inertial automorphisms on complete local rings as we shall see presently. But first, an elementary observation which lends some insight into the characteristic $p$ case:

(7) Proposition. Let $h$ be a field having characteristic $p, \neq 0$, and let $k$ be a subfield of $h$. If $k$ is perfect then the only higher derivation of $k$ into $h$ is the trivial one $Q=\left\{Q^{(i)}\right\}, Q^{(i)}$ being the zero map for $i>0$.

Proof. Let $D=\left\{D^{(i)}\right\}$ be in $3 c(k, h)$. Since $D^{(1)}\left(a^{p}\right)=p a^{p-1} D^{(1)}(a)$ $=0$, and $k=k^{p}$, it follows that $D^{(1)}$ is the zero map $\theta$. If $D^{(i)}=\theta$, for $i<n$, then, by the Leibniz rule, $D^{(n)}$ is a derivation. Thus, by the proof that $D^{(1)}=\theta$ we have $D^{(n)}=\theta$.

The following definition, due to $\mathrm{O}$. Teichmuller, is basic for our purposes. We state it for completeness and refer the reader to the algebra texts for further information [26].

(8) Definition. Let $h$ be a field having characteristic $p, \neq 0$. A subset $\mathcal{S}$ of $h$ is $p$-independent if the set of all monomials $\left\{s_{1}^{i_{1}} \cdots s_{n}^{i_{n}} \mid s_{j} \in S, i_{j}<p\right\}$ is linearly independent over $h^{p}$. If, in addition, $h^{p}(\mathcal{S})=h$ then $S$ is a $p$-basis for $h$. 
To illustrate. If $h^{p}=h$, of course, $h$ has no $p$-independent subsets. If $h^{p} \nsubseteq h$ choose $s_{1} \in h, s_{1} \notin h^{p}$. Then $\left\{s_{1}\right\}$ is a $p$-independent subset. If $h^{p}\left(s_{1}\right) \Phi h$ choose $s_{2}$ in $h$, not in $h^{p}\left(s_{1}\right)$. Then $\left\{s_{1}, s_{2}\right\}$ is a $p$-independent subset, etc.

(9) Theorem [12, p. 131, Theorem 1]. Let $\$$ be a p-basis for the subfield $k$ of a field $h$ and let $\left\{\phi_{i}\right\}$ be a sequence of maps of $S$ into $h$. There is exactly one $D$ in $\mathfrak{F}(k, h)$ such that $\left.D^{(i)}\right|_{s}=\phi_{i}\left(\left.D^{(i)}\right|_{s}\right.$ denotes the restriction of $D^{(i)}$ to $\$$ ).

A proof of Theorem 9, not the original, based on Proposition 2 and the proof of the second part of Proposition 2 can be easily summarized as follows. We note first that $S$ is an algebraically independent set over the maximal perfect subfield $k_{\infty}$ of $k$. We apply the extension, to any number of indeterminates, of the first part of Proposition 2. Thus, there is exactly one $D_{*}$ in $\mathcal{H C}\left(k_{\infty}(\delta), h\right)$ such that $D_{*}^{(i)} \mid s=\phi_{i}$. Now $k$ is separable over $k_{\infty}(\delta)$ in the linearly disjoint sense, since $S$ is a $p$-basis for both fields. In fact it is easily shown $\left[13\right.$, p. 347] that if $U$ is a basis for $k$ as a linear space over $k_{\infty}(S)$ then $U^{p}$ is also. Using the argument following Proposition 2 we conclude that each $E$ in $\mathfrak{F C}\left(k_{\infty}(S), h\right)$ has a unique extension to $\mathfrak{F}(k, h)$ and the proof is complete. We are now in a position to proceed to automorphisms.

IV. Derivation automorphisms, convergence rate automorphisms and the ramification series. Let $R$ be a complete local ring with maximal ideal $M$ and residue field $k=R / M$. Let $G$ be the group of automorphisms of $R$. The $M$ related structure of $R$ suggests a decomposition of $G$ as follows. Let $i \geq 1$.

$$
\begin{aligned}
& G_{i}=\left\{\alpha \in G \mid \alpha(a)-a \in M^{i} \text { for } a \text { in } R\right\}, \\
& H_{i}=\left\{\alpha \in G_{i} \mid \alpha(a)-a \in M^{i+1} \text { for } a \text { in } M\right\} .
\end{aligned}
$$

The series (10) is called the higher ramification series of $G$ or simply the ramification series. This

$$
G_{1} \supset H_{1} \supset G_{2} \supset H_{2} \supset \cdots
$$

series was first considered by Saunders MacLane who, in 1939, determined the invariant subrings of the groups of (10) as well as the factor groups of successive pairs of subgroups all in the case in which $R$ is the ring of integers of a $p$-adic field [30].

The $M$-adic topology on $G$ is the topology obtained by choosing, for each $\alpha$ in $G$, the cosets $\left\{G_{i} \alpha, M_{i} \alpha \mid i \geq 1\right\}$ as an open neighborhood basis at $\alpha$. One sees that $G$ is complete in the $M$-adic topology. 
A higher derivation $D=\left\{D^{(i)}\right\}$ on $R$ is said to converge if $\sum D^{(i)}$ is a convergent series of maps. This simply means that there is a sequence $\left\{n_{i}\right\}$ of nonnegative integers for which $\lim n_{i}=\infty$ and $D^{(i)}(R) \subset M^{n_{i}}, i>0$.

We consider the following subset of $\mathfrak{K C}(R) . \mathfrak{H C}_{c}(R)=\{D \in \mathfrak{H C}(R) \mid$ $\sum D^{(i)}$ converges and $\left.D^{(i)}(M) \subset M^{2}, i>0\right\}$. The succeeding list of statements is discussed below.

(i) If $D$ is in $\mathcal{K}_{c}(R)$ then $D^{(i)}(R) \subset M, i \geq 1$.

(ii) $\mathcal{H C}_{c}(R)$ is a subgroup of $\mathcal{H C}(R)$.

(iii) $\sum D^{(i)}$ is in $H_{1}$ for $D=\left\{D^{(i)}\right\}$ in $\mathfrak{F}_{c}(R)$.

(iv) $\phi: \mathcal{K C}_{c}(R) \rightarrow H_{1}$ where $\phi(D)=\sum D^{(i)}$ is a group homomorphism. The range of $\phi$ is called the group of derivation automorphisms and is denoted by $G_{D}$.

(v) $G_{D}$ is an invariant subgroup of $G$.

(vi) Given a sequence $\left\{n_{i}\right\}$ of positive integers such that $\lim n_{i}$ $=\infty$, the set $\mathcal{K C}_{\left\{n_{i}\right\}}=\left\{D \in \mathfrak{F C}(R) \mid D^{(i)}(R) \subset M^{n_{i}}, D^{(i)}(M) \subset M^{n_{i}+1}\right\}$ forms a subgroup of $\mathfrak{H}_{c}(R)$.

(vii) $\phi\left(\mathcal{F C}_{\left\{n_{i}\right\}}\right)=G_{\left\{n_{i}\right\}}$ is an invariant subgroup of $G$, called a c.r. (convergence rate) subgroup.

Proofs of (i) and (ii) are found in [18, Lemma 1, Theorem 3]. Considering (iii) and (iv), additivity of the $D^{(i)}$ and the Leibniz rule establish that $\sum D^{(i)}$ is an endomorphism. A routine check shows that $D \rightarrow \sum D^{(i)}$ is a product preserving map of $\mathcal{F}_{c}(R)$ into the endomorphism ring of $R$. But $\phi(Q)=\mathrm{Id}$. Hence $\phi(D)$ is an automorphism and is in $H_{1}$ by (i) and the fact that $D^{(i)}(M) \subset M^{2}$ for $i>0$. With reference to (v), if $\alpha$ is in $G$ and $D$ is in $\mathcal{F}_{c}(R)$, then $\alpha^{-1} D \alpha=\left\{\alpha^{-1} D^{(i)} \alpha\right\}$ is in $\mathfrak{H C}(R)$ and is, in fact, in $\mathfrak{F}_{c}(R)$ since $\alpha\left(M^{i}\right)$ $=M^{i}$ for $i \geq 0$. Also, $\alpha^{-1}\left(\sum D^{(i)}\right) \alpha=\sum \alpha^{-1} D^{(i)} \alpha$. This proves $(\mathrm{v})$ and (vii). The proof of (vi) requires only a (perhaps long) look at the definition of product in $\mathcal{H C}(R)$ and the expression

$$
\bar{D}^{(n)}=\sum\left\{(-1)^{r} D^{\left(i_{1}\right)} \cdots D^{\left(i_{r}\right)} \mid i_{1}+\cdots+i_{r}=n, i_{j}>0\right\}
$$

for the $n$th map of $\bar{D}$ the inverse of $D$.

We have now two sets of invariant subgroups of $G$, the ramification series and the c.r. subgroups. Nothing would seem to lead one to suspect that the set of c.r. subgroups is linearly ordered by inclusion. Thus one would expect that the two sets do not coincide. We shall see that this is true in general; however, we shall also see that there are relationships between the two sets. The remainder of this article is concerned primarily with these questions and with the use of derivation automorphisms to evaluate the successive factors of the ramification series (10). 
V. Approximating inertial automorphisms with derivation automorphisms. To these ends we consider next the problem of approximating inertial automorphisms with derivation automorphisms in the $M$-adic topology. We will follow, roughly, the historical development.

The first two techniques are illustrated in the case in which $R$ is a $v$-ring, that is $R$ is a complete discrete valuation ring having characteristic 0 , whereas the residue field of $R$ has characteristic $p, \neq 0$. In this case $M$ is a principal ideal. Let $\pi$ be a generator of $M$. If $\alpha$ is in $G_{i}$ then $\alpha=\operatorname{Id}+\pi^{i} \alpha^{*}$ and $\alpha$ is in $H_{i}$ if and only if $\alpha^{*}(\pi R) \subset \pi R$. Thus, if $\alpha$ is in $H_{i}, \alpha^{*}$ induces a map on $k$. Since $\alpha$ preserves sums and products we have

$$
\begin{aligned}
\alpha^{*}(a+b) & =\alpha^{*}(a)+\alpha^{*}(b), \\
\alpha^{*}(a b) & =a \alpha^{*}(b)+\alpha^{*}(a) b+\pi^{i} \alpha^{*}(a) \alpha^{*}(b) .
\end{aligned}
$$

Thus, if $\alpha \in H_{i}, \alpha^{*}$ induces a derivation $\delta_{\alpha}$ on $k, \delta_{\alpha}$ being the unique map which makes (11) commutative where $\xi$ denotes the natural map of $R$ onto $k$.

$$
\begin{aligned}
& R \stackrel{\alpha^{*} \mid}{\rightarrow} R \\
& \xi \downarrow \delta_{\alpha} \downarrow \xi \\
& k \stackrel{\delta_{\alpha}}{\rightarrow} k
\end{aligned}
$$

The homomorphism $\phi_{i}: G_{i} \rightarrow(k,+)$ and $\psi_{i}: H_{i} \rightarrow$ Der $k$ where $\phi_{i}(\alpha)=\xi \alpha^{*}(\pi)$ and $\psi_{i}(\alpha)=\delta_{\alpha}$ are basic to the process of approximating inertial automorphisms in the $M$-adic topology. The exactness of the following sequences is clear, $\epsilon$ being the natural injection.

$$
\begin{aligned}
& 0 \rightarrow H_{i} \stackrel{\epsilon}{\rightarrow} G_{i} \stackrel{\phi_{i}}{\rightarrow}(k,+), \\
& 0 \rightarrow G_{i+1} \stackrel{\epsilon}{\rightarrow} H_{i} \stackrel{\psi_{i}}{\rightarrow} \text { Der } k,
\end{aligned}
$$

We consider now the problem of constructing a pre-image in $H_{i}$ of $\delta \in$ Der $k$ with the aid of $\psi_{i}$.

Method 1. We choose $d$ in Der $R$ which induces $\delta$ as in diagram (11). We next select $i \geqq(e+1) /(p-1)$, where $p R=\pi^{e} R$, that is, $e$ is the ramification index of $R$. We also assume at this point that $p \neq 2$. Let $D=\left\{\pi^{i n} d^{n} / n !\right\}$. A check shows that $D$ is in $\operatorname{FC}(R)$. In fact, we chose $i$ large enough to insure that $D$ converges. Thus

$$
\alpha_{D}=\sum \frac{\pi^{i n} d^{n}}{n !}=\operatorname{Id}+\pi^{i}(d+\pi \cdots)
$$

is in $H_{i}$ and, apparently, $\psi_{i}\left(\alpha_{D}\right)=\delta$. 
This technique works fine for many purposes if $R$ is unramified $(e=1)$ since in that case every $\delta$ in Der $k$ lifts to $d$ in $\operatorname{Der} R[13$, p. 349, Theorem 1], $(e+1) /(p-1)=2 /(p-1) \leqq 1(p \neq 2)$, and $G_{i}=H_{i}$ for $i \geqq 1$. Thus, if $R$ is an unramified $v$-ring (ring of integers of a $p$-adic field) the above discussion implies the conclusion that $\psi_{i}$ induces an isomorphism of $H_{i} / G_{i+1}$ with Der $k$. Since $G_{i}=H_{i}, i \geqq 1$, we have all the factors of (10). As stated earlier this was first done by MacLane who used quite different methods [30].

Since, for $\alpha$ in $H_{i}, \alpha=\alpha_{1}, \bmod H_{i+1}$ where $\alpha_{1}$ is in $G_{D}$ and by iterating the above process, $\alpha=\alpha_{r}$, $\bmod H_{i+r}$, for $\alpha_{r}$ in $G_{D}$ and any $r>0$, it is simply a matter of showing that $\lim _{r} \alpha_{r}$ is in $G_{D}$ to conclude that in this case $G_{1}=H_{1}=G_{D}$.

Method 2. Given $\delta$ in Der $k$ we construct $d$ in $\mathscr{H}(k)$ so that $d^{(1)}=\delta$ (Theorem 9) and lift $d$ to $D$ in $\mathfrak{H C}(R)$ (i.e., $D^{(i)}$ induces $d^{(i)}$ for $i \geqq 1$ ). Now $\pi^{i} D=\left\{\pi^{n i} D^{(n)}\right\}$ is in $\mathfrak{K}_{c}(R)$ and

$$
\alpha_{\pi} i_{D}=\sum \pi^{n i} D^{(n)}=1+\pi^{i}\left(D^{(1)}+\pi \cdots\right)
$$

is in $H_{i}$ and clearly has the property $\psi_{i}\left(\alpha_{\pi} i_{D}\right)=\delta$.

This method is useful if $e$ and $p$ are relatively prime since in that case we have

(i) $\mathfrak{H C}(k)$ lifts to $\mathfrak{H C}(R)^{1}$ and

(ii) $H_{i}=G_{i}, i \geqq 2$.

As in the unramified case one finds that $H_{1}=G_{D}$ and that $\psi_{i}$ maps onto Der $k$. Hence $\psi_{i}$ induces an isomorphism $H_{i} / G_{i+1} \rightarrow$ Der $k$. This fact was demonstrated using other methods by the writer [17, p. 538, Theorem 5] in a paper in which it is also shown that the map $\alpha \rightarrow \xi(\alpha(\pi) / \pi)$ induces an isomorphism of $G_{1} / H_{1}$ with the group of $e$ th roots of unity in $k$. The factor group $G / G_{1}$ has also been determined in this case [16, p. 1208, Corollary 3].

The only result concerning the factors of the ramification series which encompasses all $v$-rings is due to J. Neggers [31, p. 503, Theorem 6].

(12) THEOREM. Given a v-ring $R$ with ramification $e$ and an integer $i \geqq(e+p) /(p-1)$, then, if $\alpha$ is in $G_{i}$, there is a derivation $d_{\alpha}$ on $R$ such that $\alpha^{*}=d_{\alpha}, \bmod M$.

Using the techniques of Method $1 \alpha$ in $G_{i}$ can be approximated, $\bmod G_{i+1}$, by $\beta_{i}=\sum \pi^{i n} d_{\alpha}^{i}$. Hence, $\alpha$ can be approximated, $\bmod$ $G_{i+r}$, by $\beta_{r}$ in $G_{D}$ for any given integer $r$. One observes that $\beta=\lim _{r} \beta_{r}$ is in $G_{D}$ and, hence,

\footnotetext{
${ }^{1}$ Statement (i) was proved by the writer for $R$ unramified [14, p. 579, Corollary 1$]$. The result is easily extended to tamely ramified $v$-rings.
} 
(13) Corollary. $G_{D} \supseteq G_{i}$ for $i \geqq(e+p) /(p-1)$.

The next corollary is also an immediate consequence of Neggers' Theorem. Let $\operatorname{Der}_{\mathrm{I}} R=\{d \in \operatorname{Der} R / d(\pi) \in \pi R\}$. The homomorphisms $\Lambda_{i}: G_{i} \rightarrow \operatorname{Der} R / \pi$ Der $R, \quad \Lambda_{i}^{\prime}: H_{i} \rightarrow \operatorname{Der}_{I} R / \pi$ Der $R$

and

$$
\Lambda_{i}^{*}: G_{i} \rightarrow \operatorname{Der} R / \operatorname{Der}_{\mathrm{I}} R
$$

are defined by mapping $\alpha$ into the coset of $d_{\alpha}$.

(14) CoRollary. Given that $i \geqq(e+p) /(p-1)$ the following are exact.

$$
\begin{aligned}
& 0 \rightarrow G_{i+1} \stackrel{\epsilon}{\rightarrow} G_{i} \stackrel{\Lambda_{i}}{\longrightarrow} \text { Der } R / \pi \text { Der } R \rightarrow 0, \\
& 0 \rightarrow H_{i} \stackrel{\epsilon}{\rightarrow} G_{i} \stackrel{\Lambda_{i}^{*}}{\longrightarrow} \text { Der } R / \operatorname{Der}_{\mathrm{I}} R \rightarrow 0, \\
& 0 \rightarrow G_{i+1} \stackrel{\epsilon}{\rightarrow} H_{i} \stackrel{\Lambda_{i}^{\prime}}{\longrightarrow} \operatorname{Der}_{\mathrm{I}} R / \pi \text { Der } R \rightarrow 0 .
\end{aligned}
$$

The last sequence above states that the range of $\psi_{i}$ is precisely the subgroup of those $\delta$ in Der $k$ which lift to Der $R$. Neggers also showed that $\operatorname{Der} R=\operatorname{Der}_{I} R$ if and only if every $\delta \in \operatorname{Der} k$ lifts to $\operatorname{Der} R[31$, p. 500 , Corollary 1 ].

The factors of the ramification series of an unramified complete regular local ring $R$ (i.e. a power series ring in $n$ indeterminates over a field or an unramified $v$-ring) have been determined by the writer $[15$, p. 37 , Theorems $2.1,2.2,2.3]$. It is implicit in this work that $H_{1}=G_{D}$ for such a ring $R$.

The following assertion regarding c.r. groups does not appear in the literature. It is easily proved. One might regard it as an initial result from which one would proceed to study c.r. groups in case $R$ is an unramified complete regular local ring.

(15) Proposition. Let $R$ be an unramified complete regular local ring and let $\left\{n_{i}\right\}$ be a sequence of positive integers for which $\lim _{i} n_{i}=\infty$ and $n_{r} \leqq n_{i}+n_{r-i}$ for all $r$ and $i<r$. Then $H_{t}=G_{\left\{n_{i}\right\}}$ where $t=\min _{i} n_{i}$.

VI. A more versatile method of approximating. In order to obtain further results a more versatile method of constructing derivation automorphisms would seem to be needed. Such a method is supplied by the following theorem for which we assume that $R$ is a complete regular local ring in the unequal characteristic case. I. S. Cohen showed that $R$ contains an unramified $v$-ring $V$ having the property $V+M / M=k[3, \mathrm{p} .79$, Theorem 11]. 
(16) Theorem [18, p. 38, TheOREM 4]. Let $\$ \subset V$ be a set of representatives of a p-basis $\bar{\delta}$ of $k$. Given a sequence $\left\{\phi_{i}\right\}$ of maps of $s$ into $R$, there is exactly one $D$ in $\operatorname{FC}(V, R)$ for which $\left.D^{(i)}\right|_{s}=\phi_{i}, i \geqq 1$. Also $D^{(i)}(V) \subseteq M^{n_{i}}, i \geqq 1$, if and only if $D^{(i)}(\delta) \subseteq M^{n^{n}}, i \geqq 1$.

As an immediate corollary we have the following which has been referred to earlier:

(17) Corollary. $\mathfrak{H}(k)$ lifts to $\mathfrak{H}(V)$.

The proof of Theorem 16 follows the pattern of argument used to establish Theorem 9 as follows. Let $V_{0}$ be the (unique) sub $v$-ring of $V$ with residue field $k_{\infty}$, the maximal perfect subfield of $k$. We note first that the only derivation of $V_{0}$ into $R$ is the zero map and, hence, as in the proof of Proposition $7, \mathfrak{H}\left(V_{0}, R\right)$ contains only the trivial higher derivation. Since $\bar{\delta}$ is algebraically independent over $k_{\infty}, \mathcal{S}$ is algebraically independent over $V_{0}$. Thus, proceeding as in the proof of Theorem 9 , we can define $D_{*}$ on $V_{0}[\delta]$ by the condition $\left.D_{*}^{(i)}\right|_{\delta}=\phi_{i}$, $i \geqq 1$. A set $U$, of representatives of a linear basis $\bar{U}$ of $k$ over $k_{\infty}(\bar{\delta})$, is chosen in $V$. For any preassigned integer $n, V$ is a free module over $V_{0}[\delta]$, modulo $p^{n} V$, with free generators the set $U p^{n}$. This fact implies that $D_{*}$ has a unique extension to $D$ in $\mathscr{K}(V, R)$, again, as in the proof of Theorem 9 though admittedly the details here are a little messy.

It was proved by I. S. Cohen that if $R$ is as in Theorem 16 then $R$ has the form $S[\pi]$ where $S=V\left[\left[X_{1}, \cdots, X_{n}\right]\right]$ is a power series ring in $n$ indeterminates over $V$ and $\pi$ is algebraic over $S$ having a minimal polynomial over $S$ of a particular kind called an Eisenstein polynomial [3, p. 92, Theorem 17]. Thus, the potential usefulness of Theorem 16 for the construction of derivation automorphisms on such rings is enhanced by the fact that one knows how $D$ in $\mathfrak{H C}(V, R)$ extends to a higher derivation defined on $R$. Theorem 16 has been used in the study of the inertial automorphism group of $v$-rings with ramification $p$ by the writer [19] and Martin N. Heinzer [24] and for $v$-rings with ramification $2 p$ by Robert D. Davis [4].

VII. Some applications and some problems. For $R$ an unramified complete regular local ring the factors $H_{i} / G_{i+1}$ are all direct sums of copies of Der $k$, the number of summands depending on $i$ and the dimension of $R$. The factors $G_{i} / H_{i}$ are all zero, or, in the equal characteristic case, they are direct sums of copies of $k^{+}$, the additive group of $k$, the number of summands determined again by $i$ and the dimension of $R[15, \mathrm{p} .37$, Theorems $2.1,2.2,2.3]$. Also, as we have 
observed before $G_{D}=H_{1}$, and according to Proposition 15 a large class of c.r. groups, defined by a natural condition on the convergence rate, coincide with the subset $\left\{H_{i}\right\}_{i \geq 1}$ of the ramification series. All of this changes if $R$ is ramified. In particular, if $R$ is a $v$-ring with ramification $p$ then, in general, $G_{D}$ is not a term of the ramification series. Moreover, the factors of the ramification series, exhibit a good bit of variety, and are no longer dependent on $k$ (and the dimension of $R$ ) [19, p. 46, Theorem 2]. As an initial investigation into the c.r. groups $M$. Heinzer has determined the relationship between $G_{D}, G_{\{i\}}$ and the ramification series, again, for the v-ring $R$ with ramification $p$ [24]. If $R$ is tamely ramified $G_{\{i\}}=G_{D}$, a fact which is implied by the application of Method II of this paper for approximation of inertial automorphism since all automorphisms constructed by Method II are in $G_{\{i\}}$.

Heinzer found that the relationship between $G_{\{i\}}$ (there denoted $G_{S}$ ) and the other groups was described by a number of different cases [24, see table]. Always $H_{1} \supset G_{\{i\}} \supset H_{2}$. Generally, $G_{\{i\}}$ is different from $G_{D}$ and is not a term of the ramification series.

Robert D. Davis [4] carried out the complete analysis of the factors of the ramification series (except $G / G_{1}$ ) for the general $v$-ring of ramification $2 p$, using a modification of the procedures used by the writer in the case $e=p$ [19]. Davis found that eight different pairs of factor groups $H_{1} / G_{2}$ and $H_{2} / G_{3}$ occur. However, for $i>2, H_{i} / G_{i+1}$ is the group of $\delta$ in Der $k$ which lifts to Der $R$ (see Neggers result, Theorem 12) and is always given by $\left\{\delta \in \operatorname{Der} k \mid \delta \xi\left(\pi^{2 p} / p\right)=0\right\}$. Considerable variety was also found in the factors $G_{i} / H_{i}$ because of the possible presence of Galois maps, that is automorphisms of finite order. Such maps $\alpha$ always occur in a $G_{i}-H_{i}$ gap, i.e., $\alpha \in G_{i}, \alpha \notin H_{i}$.

Certain conclusions can be drawn from the results discussed above. For example, the problem of obtaining a complete analysis of the factors of the ramification series of all $v$-rings having a given ramification $e$, a problem which has been solved for the cases $(e, p)=1, e=p$, and $e=2 p$, is apparently too complex to be manageable in the remaining cases. This is suggested by Davis' results for $e=2 p$. A further classification of $v$-rings is needed in order to divide the problem into manageable parts.

At this point very little is known about the c.r. groups other than Heinzer's analysis of $G_{\{i\}}$ and Proposition 15. For example, by Proposition 15, the set of groups $G_{\left\{n_{i}\right\}}$ for which $n_{r} \leqq n_{i}+n_{r-1}$ is totally ordered by inclusion, if $R$ is unramified and regular. It would be surprising if this were true in general.

Another interesting question is the following: Given distinct se- 
quences $\left\{n_{i}\right\}$ and $\left\{m_{i}\right\}$ of positive integers such that $\lim _{i} n_{i}=\lim _{i} m_{i}$ $=\infty$. Is there a complete local ring $R$ for which $G_{\left\{n_{i}\right\}} \neq G_{\left\{m_{i}\right\}}$ ? Also, what property of $R$ determines whether or not $G_{\left\{n_{i}\right\}}=G_{\left\{m_{i}\right\}}$ ? These questions are of particular interest when $\left\{n_{i}\right\}$ and $\left\{m_{i}\right\}$ differ very little, e.g., $n_{i}=m_{i}$ for $i \neq p$ and $n_{p}=m_{p}+1$. The analysis is likely to be quite straightforward if one restricts attention to unramified regular rings only. However, the answers to questions such as these may give rise to interesting classifications of wildly ramified $v$-rings.

Another result which would be of interest is a generalization of Neggers' theorem (Theorem 12) to complete regular local rings of dimension greater than one.

\section{BIBLIOGRAPHY}

1. R. Baer, Algebraische Theorie der differentierbaren Funktionenkorper, S.-B. Heidelberger Akad. Wiss. Abh. 8 (1927), 15-32.

2. R. Berger, Differential höherer Ordnung und Körpererweiterungen bei Prizahlcharakteristik, S.-B. Heidelberger Akad. Wiss. Math.-Natur. Kl. 1966, 143-202. MR 34 \#2570.

3. I. S. Cohen, On the structure and ideal theory of complete local rings, Trans. Amer. Math. Soc. 59 (1946), 54-106. MR 7, 509.

4. R. D. Davis, On the inertial automorphisms of a class of ramified v-rings, Dissertation, Florida State University, Tallahassee, Fla., 1969.

5. R. L. Davis, A Galois theory for a class of purely inseparable field extensions, Dissertation, Florida State University, Tallahassee, Fla., 1969.

6. - A Galois theory for a class of purely inseparable exponent two field extensions, Bull. Amer. Math. Soc. 75 (1969), 1001-1004. MR 39 \#5524.

7. M. Gerstenhaber, On the deformation of rings and algebras, Ann. of Math. (2) 79 (1964), 59-103. MR 30 \#2034. \#142.

8. - On modular field extensions, J. Algebra 10 (1968), 478-484. MR 38

9. H. Hasse, Theorie der hoheren Differentiale in einem algebraischen Funktionenkorper mit vollkommenen Konstantenkorper bei beliebiger Charakteristik, J. Reine Angew. Math. 175 (1936), 50-54.

10. H. Hasse and F. K. Schmidt, Noch eine Begrundung der Theorie der hoheren Differentialquotienten in einem algebraischen Funktionenkorper einer Unbestimmten, J. Reine Angew. Math. 177 (1936), 215-237.

11. N. Heerema, Derivations and embeddings of a field in its power series ring, Proc. Amer. Math. Soc. 11 (1960), 188-194. MR 23 \#A93.

12. - Derivations and embeddings of a field in its power series ring. II, Michigan Math. J. 8 (1961), 129-134. MR 25 \#69.

13. — Derivations on p-adic fields, Trans. Amer. Math. Soc. 102 (1962), 346-351. MR $26 \# 1311$.

14. —— Embeddings of a p-adic field and its residue field in their power series rings, Proc. Amer. Math. Soc. 14 (1963), 574-580. MR 27 \#1472.

15. - Derivations and automorphisms of complete regular local rings, Amer. J. Math. 88 (1966), 33-42. MR 33 \#5665.

16. —, Equivalence of tamely ramified v-rings, Proc. Amer. Math. Soc. 17 (1966), 1207-1210. MR 34 \# 7502. 
17. - Inertial isomorphisms of v-rings, Canad. J. Math. 19 (1967), 529-539. MR $35 \# 1588$.

18. - Convergent higher derivations on local rings, Trans. Amer. Math. Soc. 132 (1968), 31-44. MR 36 \#6406.

19. - Inertial automorphisms of a class of wildly ramified v-rings, Trans. Amer. Math. Soc. 132 (1968), 45-54. MR 36 \#6407.

20. - Exponential automorphisms on complete local rings, Trans. Amer. Math. Soc. (to appear).

21. - - An extension of classical Galois theory to inseparable fields (to appear).

22. - A group of a Lie algebra, J. Reine Angew. Math. (to appear).

23. M. N. Heinzer, Higher derivations of wildly ramified v-rings, Proc. Amer. Math. Soc. 23 (1969), 94-100. MR 39 \#5553.

24. - - Strongly convergent derivation automorphisms on a class of wildly ramified v-rings, Duke Math. J. (to appear).

25. N. Jacobson, Galois theory of purely inseparable fields of exponent one, Amer. J. Math. 66 (1944), 645-648. MR 6, 115.

26. - Lectures in abstract algebra. Vol. III: Theory of fields and Galois theory, Van Nostrand, Princeton, N.J., 1964. MR $30 \# 3087$.

27. - Lie algebras, Interscience Tracts in Pure and Appl. Math., vol. 10, Interscience, New York, 1962. MR $26 \# 1345$.

28. Arno Jaeger, Eine Algebraische Theorie vertauschbarer Differentiationen für Körper beliebiger Charakteristik, J. Reine Angew. Math. 190 (1952), 1-21. MR 14, 130.

29. P. Leroux and P. Ribenboim, Dérivations d'ordre supérieur dans les catégories semi-additives (to appear).

30. Saunders Mac Lane, Subfields and automorphism groups of p-adic fields, Ann. of Math. (2) 40 (1939), 423-442.

31. J. Neggers, Derivations on F-adic fields, Trans. Amer. Math. Soc. 115 (1965), 496-504. MR 33 \#5610.

32. J. B. Miller, Homomorphisms, higher derivations and derivations on associative algebras, Acta. Sci. Math. (Szeged) 28 (1967), 221-231. MR 35 \#2927.

33. G. Paulus, Inseparabilitat und Differentiation in Korpern der Charakteristik $p$, Zulassungsarbeit, Heidelberg, 1954.

34. P. Ribenboim, Algebraic theory of higher order derivations, Trans. Roy. Soc. Canad. (to appear).

35. M. E. Sweedler, Structure of inseparable extensions, Ann. of Math. (2) 87 (1968), 401-410. MR 36 \#6391.

36. - Correction to: "Structure of inseparable extensions," Ann. of Math. (2) 89 (1969), 206-207. MR 38 \#4451.

37. O. Teichmüller, Differentialrechnung bei Charakteristik p, J. Reine Angew. Math. 175 (1936), 89-99.

38. M. Weisfeld, Purely inseparable extensions and higher derivations, Trans. Amer. Math. Soc. 116 (1965), 435-449. MR 33 \#122.

39. E. Wishart, Higher derivations on p-adic fields, Dissertation, Florida State University, Tallahassee, Fla., 1965.

40. O. Zariski and P. Samuel, Commutative algebra. Vols. 1, 2, University Series in Higher Math., Van Nostrand, Princeton, N.J., 1957, 1960. MR 19, 833; MR 22 \#11006.

41. F. Zerla, Iterative higher derivations in fields of prime characteristic, Michigan Math. J. 15 (1968), 407-415. MR 39 \#185. 